\title{
OCT in Dermatology
}

\author{
J. Welzel, E. Lankenau, G. Hüttmann, and R. Birngruber
}

\subsection{Technical Demands for a Skin OCT-System}

\subsubsection{Introduction (OCT-Systems in Dermatology)}

In all OCT-systems used in dermatology, the intrinsic interferometer required for the depth-resolution (A-scan) is realized in a mono-mode fiber technology (Fig. 35.1). For the so-called time domain OCT (TD-OCT) (Fig. 35.1a), different techniques of moving the reference arm are established. The optical length of the reference arm can be varied by moving mirror systems as well as by fibre stretching. For an optimal axial resolution, the A-scan is synchronized by moving the focus inside the tissue as realized in the SkinDex300 (Isis Optronics GmbH, Germany). Other systems measure without moving the probe focus relative to the tissue, which results in an easier instrumentation, a faster scanning rate, but compromises the lateral resolution. Since 2003, OCT device based on the so-called spectral domain OCT (SD-OCT) (Fig. 35.1b), in which the reference arm has a static position and the interference modulation, detected by a spectrometer, is used to generate the depth information of the A-scan ("Spectral Radar OCT"). The main advantages of this technique are an order of magnitude faster scanning rate and an up to $10 \mathrm{~dB}$ higher contrast. On the other side, the SD-OCT is phase sensitive, which means that the depth information has to be recorded very fast in order not to destroy the interferometric signal.

The newest OCT-method is the so-called swept source OCT (SS-OCT) $[1$, 2] (Fig. 35.1c). Instead of a broad-band light source, SS-OCT is based on a fast tunable laser. In contrast to SD-OCT, the depth information is not registered by a spectrometer but by a photodiode synchronized with the tunable laser source. The advantages of SS-OCT are potentially a higher power and a faster measurement speed, depending on the laser technology available.

The axial resolution generelly depends on the bandwidth of the light source. New developments of broadband superluminescent diodes and lasers enable OCT-systems with an axial resolution down to $1 \mu \mathrm{m}$ [3-5]. Up to now 

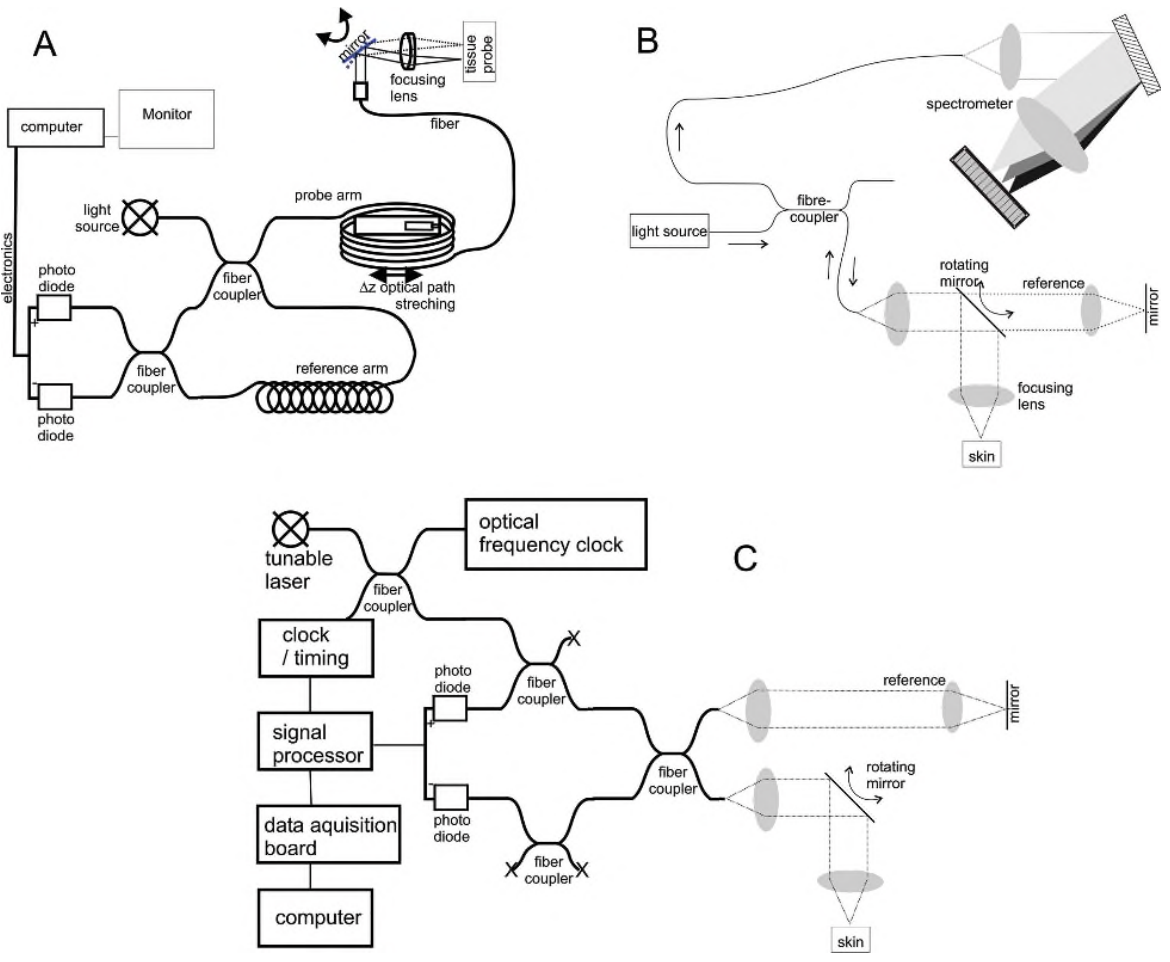

Fig. 35.1. Principles of the three different fiber-based OCT-techniques for depth detection in dermatology: an infrared light source is coupled into a single mode fiber system and splitted into a reference and probe arm. The backscattered light from the probe is superposed with the reference light and detected by photodiodes $(\mathbf{a}, \mathbf{c})$ or a spectrometer (b). Time Domain OCT (a), Spectral Domain OCT (b), and swept source OCT (c)

those high-resolution OCT-systems are investigated in numerous experimental and clinical studies and will certainly result in new commercial intruments in the future.

To generate a two- or three-dimensional image, one or two lateral scanning systems are required. There are some conventional OCT scanners for dermatological purposes, which are available commercially (SkinDex 300, ISIS optronics GmbH, Mannheim, Germany; Imalux Niris Imaging System, IMALUX, Cleveland, Ohio, USA). Experimental OCT devices are built by several research institutes and are adapted for dermatologic questions (i.e., spectral high-resolution OCT, Thorlabs HL AG, Lübeck, Germany). In most cases, the instruments are compact and easy to use (Fig. 35.3b). Table 35.1 gives an overview of different OCT specifications currently used in dermatology (the list may not be complete). 
Table 35.1. Specifications of different OCT-systems in dermatology

\begin{tabular}{|c|c|}
\hline $\begin{array}{l}\text { Producer } \\
\text { (examples) }\end{array}$ & $\begin{array}{r}\text { TD- } \\
\text { MLL GmbH, } \\
\text { Lübeck, } \\
\text { Germany }\end{array}$ \\
\hline $\begin{array}{l}\text { Central wavelength }(\mathrm{nm}) \\
\text { Axial resolution } \\
\text { Speed/A-scan }\end{array}$ & $\begin{array}{l}830 \quad 1,310 \\
\sim 15 \mu \mathrm{m} \\
20 \mathrm{~Hz}\end{array}$ \\
\hline
\end{tabular}

(a)

\begin{tabular}{|c|c|c|}
\hline -OCT & SD-OCT & SS-OCT \\
\hline $\begin{array}{c}\text { ISIS } \\
\text { optronics } \\
\text { GmbH, } \\
\text { Mannheim, } \\
\text { Germany }\end{array}$ & $\begin{array}{l}\text { Thorlabs HL } \\
\text { AG, Lübeck, } \\
\text { Gemany }\end{array}$ & $\begin{array}{c}\text { Thorlabs } \\
\text { Inc. } \\
\text { USA }\end{array}$ \\
\hline $\begin{array}{l}1,310 \\
\sim 5 \mu \mathrm{m}\end{array}$ & $\begin{array}{cc}830 & 930 \\
\sim 10 \mu \mathrm{m} & \sim 4 \mu \mathrm{m}\end{array}$ & $\begin{array}{l}1,325 \\
\sim 7 \mu \mathrm{m}\end{array}$ \\
\hline, $50 \mathrm{~Hz}$ & $1.4 \mathrm{kHz}$ & $16 \mathrm{kHz}$ \\
\hline
\end{tabular}

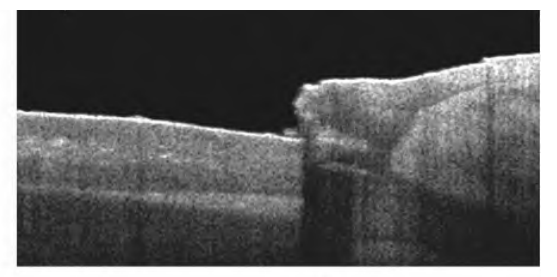

(b)

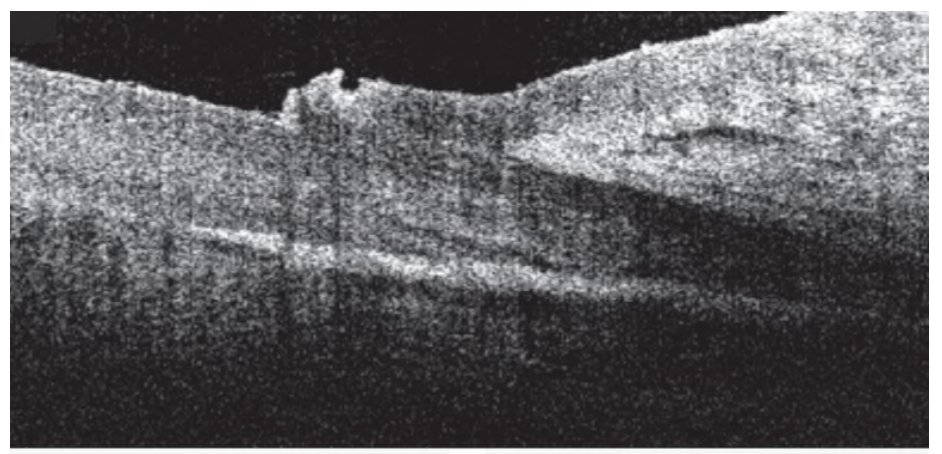

(c)

Fig. 35.2. Fingernail $\left(4 \times 2 \mathrm{~mm}^{2}\right)$. (a) TD-OCT with a resolution of about $15 \mu \mathrm{m}$, central wavelength $830 \mathrm{~nm}$. (b) SD-OCT with a resolution of about $5 \mu \mathrm{m}$, central wavelength $830 \mathrm{~nm}$. (c) SS-OCT central wavelength 1,300 nm

To demonstrate the potential of the different OCT techniques, Fig. 35.2 shows cross-sectional images of a fingernail with three different OCT systems. The fixed probe focus is positioned close to the surface of the tissue.

The images of TD-, SD-, and SS-OCT look similar in Fig. 35.2. The finger is laterally scanned from the nail (left side) over the nail fold to the skin (right side). The uppermost tissue layer on the right side is the epidermis followed by the dermis. Below the skin the finger nail can be distinguished. The higher intense signal below the nail describes the nail matrix. The best contrast 
of the nail matrix is measured by SS-OCT (Fig. 35.2c). The best contrast of the border between epidermis and dermis is measured by SD-OCT. The penetration depth of the three measurements is comparable. With a measurement resolution smaller than $7 \mu \mathrm{m}$ the pixel size (speckle size) becomes smaller, which leads to a better contrast of small tissue structures inside the tissue (Fig. 35.2b, c).

\subsubsection{Medical Applicators}

In a more confocal OCT realization, the lateral resolution is increased by strong focusing lens systems. To obtain information on deeper areas of the tissue, which are out of focus, the lenses must be moved in $z$-direction during depth scanning. In contrast to confocal microscopy, the measurement depth is higher because of the interferometric amplification of the backscattered light from tissue depth. The focus movement has to be synchronous to the optical path variation of the reference light. Beside a high resolution OCT imaging of the morphological structures of the skin, optical parameters like scattering coefficient $\mu_{\mathrm{s}}$, absorption coefficient $\mu_{\mathrm{a}}$, refractive index RI, and mean scattering angle MSA can be calculated [6]. Localized measurements of this optical parameters can provide additional information, and may increase the clinical potential of OCT by allowing quantitative discrimination between different tissue types. The measurement of the optical parameters can quantify the influence of treating the skin with different pharmaceutical products.

The disadvantage of such a dermatology applicator is that the skin has to be measured in index-matched contact. There are different skin diseases like wound healing where an index matching solution is difficult to use. Because the moving lense system inside the applicator is relatively large, the handling is complicated (Fig. 35.3a). Finally, this OCT-applicator can only be used in combination with TD-OCT.

In most clinically used probes and catheters, the optical components of the sample arm are fixed. The OCT applicator has to be small and easy to

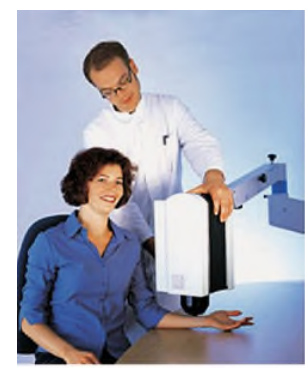

(a)

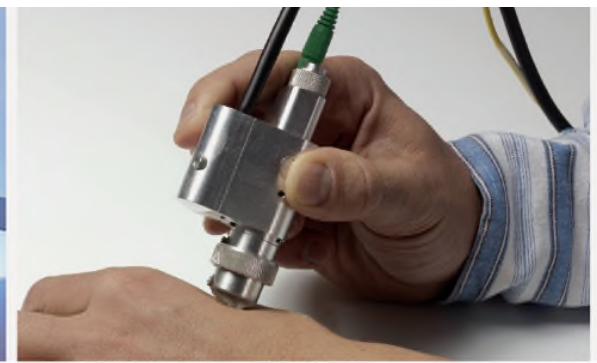

(b)

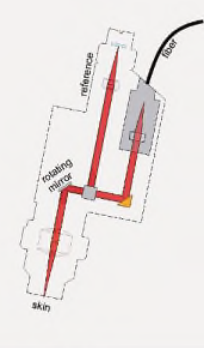

(c)

Fig. 35.3. (a) SkinDex300, ISIS optronics GmbH, Germany. (b) Small OCTapplicator for use in dermatology, Thorlabs HL AG, Germany. (c) Principle setup of $(\mathbf{b})$ 

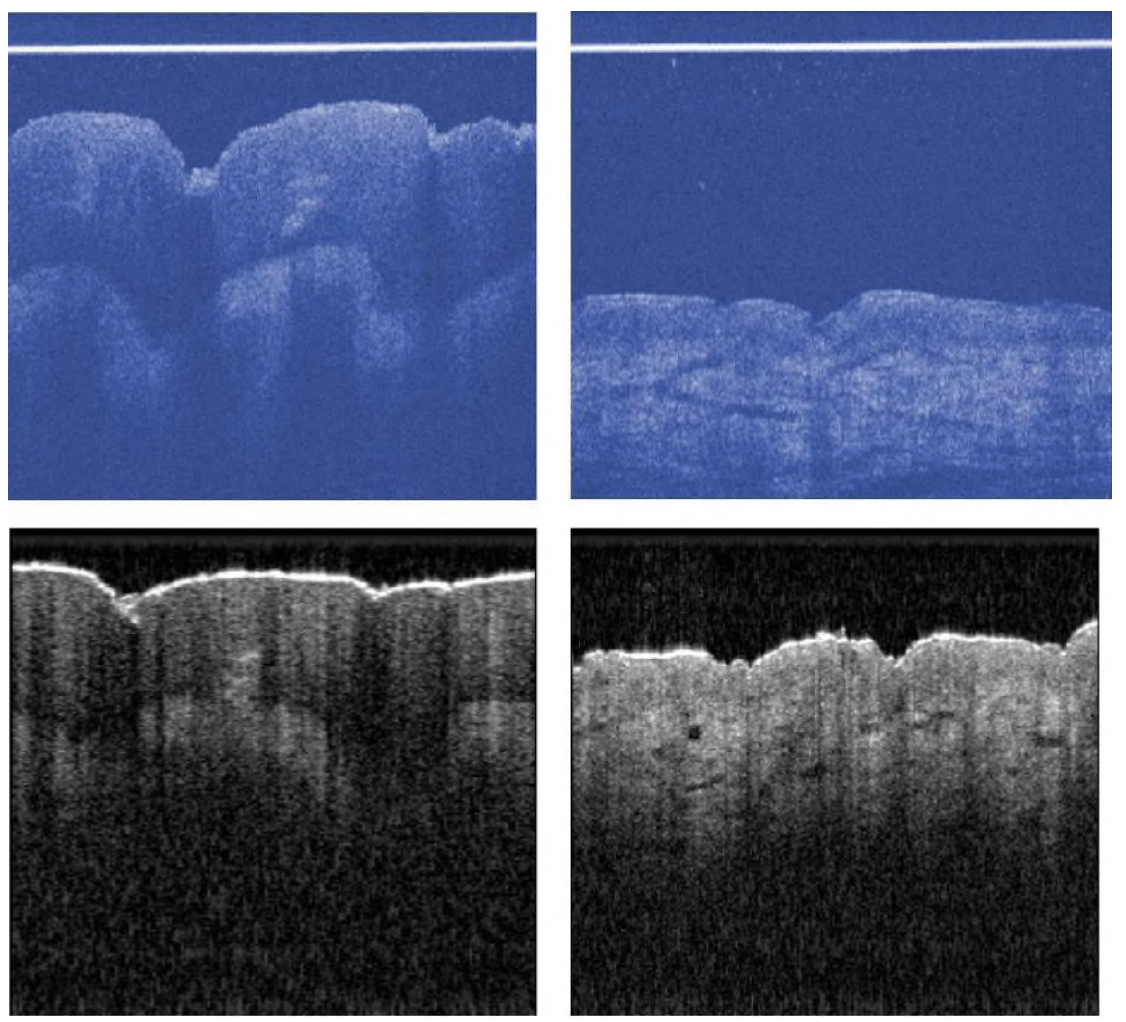

Fig. 35.4. OCT images of human skin. (Top) SkinDex 300, ISIS Optronics; 1,300 nm OCT; $1 \times 0.9 \mathrm{~mm}^{2}$; specified resolution, $5 \mu \mathrm{m}$ axial and $3 \mu \mathrm{m}$ lateral. (Bottom) High Resolution OCT $830 \mathrm{~nm} ; 2 \times 0.9 \mathrm{~mm}^{2}$; resolution, $4 \mu \mathrm{m}$ axial and approx. $8 \mu \mathrm{m}$ lateral. Left, finger tip; Right, forearm skin

handle (Fig. 35.3b). Therefore, most users abandon a moving of focus inside the probe.

For example, Fig. 35.4 images the difference between OCT measurements with a synchronously moving focus inside the tissue (upper images) and with a fixed focus positioned at the surface of the skin (images at the bottom). The tissue structures by moving the focus are better resolved (top of Fig. 35.4). The upper white line describes the border to the index matched glass plate. The lower images in Fig. 35.4 are measured without contact through air. So the signal from the skin surface is relatively high.

\subsubsection{Quantification of Tissue Parameters}

By balancing the advantages and disadvantages of the different OCT systems, it is very important that a quantification of the tissue consistence is possible. As described by Knüttel et al. [6], there is an exact theory to calculate the 


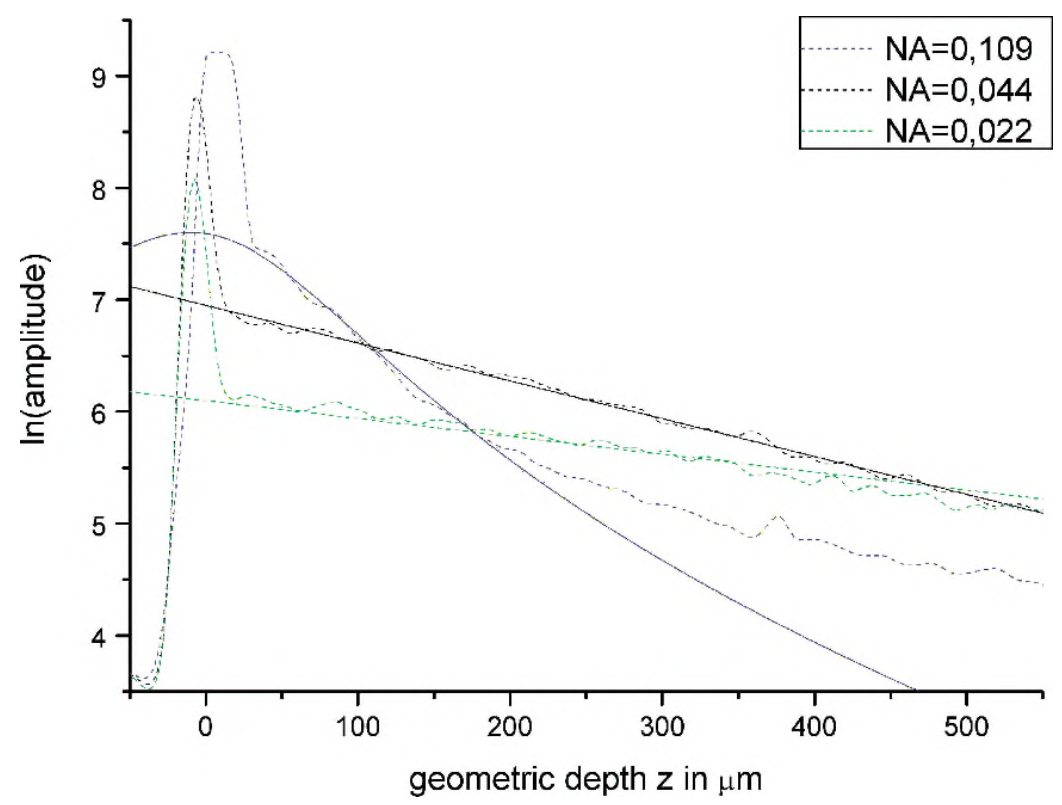

Fig. 35.5. TD-OCT with a central wavelength of $830 \mathrm{~nm}$. Averaged A-scan of a solid isotropic scatterer $\left(\mu_{\mathrm{s}}=10 \mathrm{~cm}^{-1}\right)$ measured at different numerical aperture. The focus is fixed on the surface of the probe. The high signal at depth zero comes from the jump of the refractive index (air to probe). The measured curves are fitted by eq. (35.4) (solid lines)

optical tissue parameters by using a moving focus OCT-system. Besides a high resolution OCT imaging of the morphological structures of the skin, optical parameters like scattering coefficient $\mu_{\mathrm{s}}$, absorption coefficient $\mu_{\mathrm{a}}$, refractive index RI, and mean scattering angle MSA can be calculated [6]. Measuring with the fixed focus OCT, it is possible to quantify the tissue by the attenuation coefficient using a simple theory. Figure 35.5 shows averaged A-scans of a light scattering probe. The probe focus is positioned at the surface.

For a high numerical aperture (here, e.g., 0.109, which is equal to a focus diameter of about $10 \mu \mathrm{m}$ ) and a fixed focus, the decrease of the logarithmic signal is not linear inside a scattering probe as expected from the LambertBeer law, (blue line in Fig. 35.5). The main intensity comes from the focus area and the penetration depth is decreased. There are two ways to calculate the optical attenuation. One possibility is to move the focus synchronously to the optical path variation of the reference light as described earlier. Otherwise, it is necessary to know the theoretical dependence of the OCT signal on the depth. In addition to the knowledge of the point spread function (which depends on the numerical aperture) and the exponential Lambert-Beer law (which depends on the attenuation coefficient of the probe), it is necessary to include an exact theory of light scattering which includes the effects of multiple light scattering inside the tissue. This is beyond the scope of this article. 
For nearly parallel light (low NA), the optical attenuation parameter $\mu_{\mathrm{t}}=$ $\mu_{\mathrm{a}}+\mu_{\mathrm{s}}$ of the probe will be calculated directly from the slope of the measured logarithmic A-scan (green line in Fig. 35.5). The lateral OCT resolution is poor but for some clinical applications the lateral resolution is not that important. For example, there are different areas of the skin where the thickness of the different tissue layers is constant.

In normal clinical use and for easy handling (Fig. 35.4b) it is possible to make a compromise. An alternative is a larger spot size, black line in Fig. 35.3, with a less focused beam which remains sufficiently sharp over the entire imaging depth at the expense of resolution. For a focus at the surface of the probe, the logarithmic decrease of the backscattered light is nearly linear. An attenuation coefficient can be calculated [7]. Relative changes of the attenuation coefficient $\mu_{\mathrm{t}}=\mu_{\mathrm{a}}+\mu_{\mathrm{s}}$ quantify the changes of optical parameters of the skin. The propagation of light inside the tissue is influenced by both scattering and absorption, although the latter is much lower in the near infrared. Therefore, the slope of the attenuation function of the backscattered light depends mainly to the scattering coefficient $\left[\mu_{\mathrm{s}}\right]$, which is the probability of a photon being backscattered per tissue depth.

As described by Faber et al. [7], $\mu_{\mathrm{t}}$ can be calculated by the knowledge of the used Rayleigh length $z_{0}$. For each OCT system, the Rayleigh length has to be measured exactly. This theory is also described in [8]: For a numerical aperture smaller than 0.08 and $\mu_{\mathrm{t}}<60 \mathrm{~cm}^{-1}$ [7], it is possible to approximate following formulas.

The confocal point spread function in OCT is given by

$$
I_{\mathrm{PSF}}(z)=\frac{I_{0}}{1+\left(\frac{z-z_{\mathrm{cf}}}{\alpha z_{0}}\right)^{2}} .
$$

The Rayleigh length $z_{0}$ is given by the used numerical aperture. $z_{\mathrm{cf}}$ is the relative focus position to the surface of the probe. As described in [7], $\alpha=2$ inside a scattering probe. $I_{0}$ is the normalized probe intensity.

The intensity decrease for single backscattered photons inside the scattering tissue can be described by the Lambert-Beer law:

$$
I_{\text {tissue }}(z)=I_{0} \mathrm{e}^{-2 \mu_{\mathrm{t}} z} .
$$

The measured modulation amplitude is proportional to

$$
A(z) \propto \sqrt{I_{\text {ref }} I_{\text {probe }}(z)} \propto \mathrm{e}^{-2 \mu_{\mathrm{t}} z} \sqrt{\frac{1}{1+\left(\frac{z-z_{\mathrm{cf}}}{\alpha z_{0}}\right)^{2}}},
$$

where $I_{\text {ref }}$ is the constant intensity of the reference light and $I_{\text {probe }}=$ $I_{\mathrm{PSF}} I_{\mathrm{tissue}}$. Therefore, the measurements in Fig. 35.3 are fitted by (35.4) [8]:

$$
\ln (A(z))=-2 \mu_{\mathrm{t}} z-\frac{1}{2} \ln \left(1+\left(\frac{z-z_{\mathrm{cf}}}{\alpha z_{0}}\right)^{2}\right)+C .
$$


$C$ is a constant that depends on the power of light source and $z_{\mathrm{cf}}$ was set to zero in Fig. 35.3, because the focus was positioned at the surface of the probe.

Implementation of this model for existing OCT systems will be straightforward and enables a quantification of tissue condition. Localized quantitative measurement of the attenuation coefficient of different tissues can significantly improve the clinical value of OCT [9].

\subsubsection{Image Processing}

For skin diagnosis it is necessary to analyze the OCT images. If the skin is measured without index-matched contact, the analyzing software has to find first the surface of the skin. In Fig. 35.6 this surface detection is imaged as

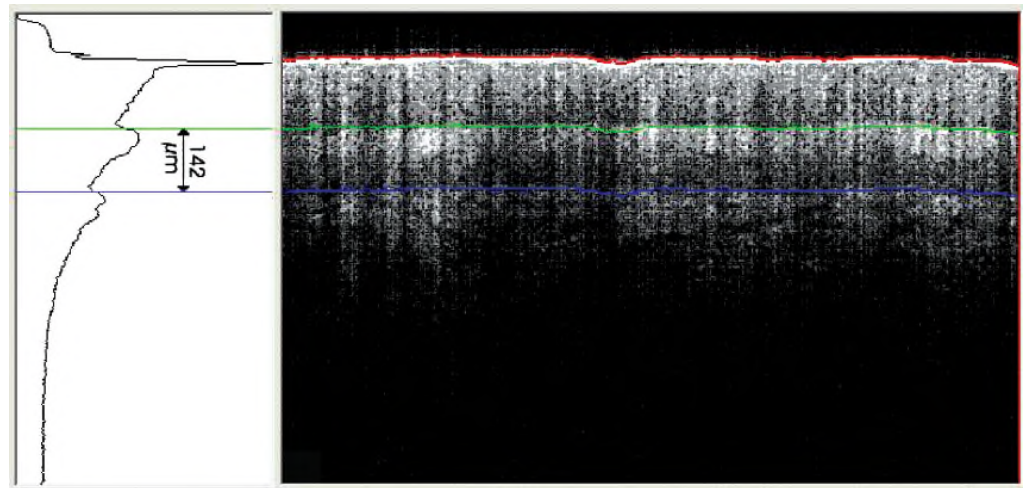

(a)

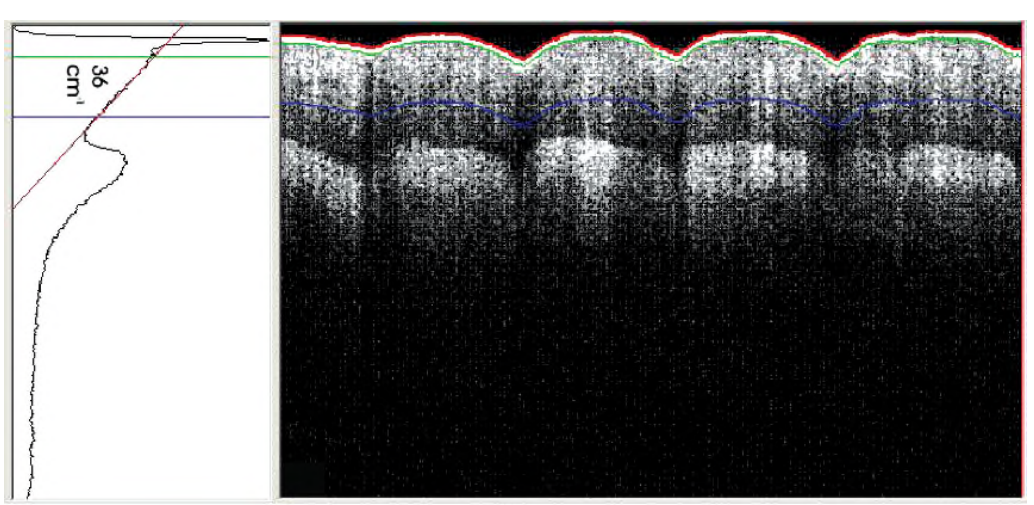

(b)

Fig. 35.6. (a) (Right) SD-OCT $(\lambda=930 \mathrm{~nm})$ of inner side at the hand $\left(2 \times 1.1 \mathrm{~mm}^{2}\right)$ with surface identification (red line). (Left) Averaged A-scan: thickness of a layer (epidermis without stratum corneum) measured between green and blue line. (b) (Right) SD-OCT $(\lambda=930 \mathrm{~nm})$ of a fingertip $\left(2 \times 1.1 \mathrm{~mm}^{2}\right)$ with surface identification (red line). (Left) Averaged A-scan: attenuation coefficient of a layer (upper stratum corneum) fitted between green and blue line 
red surface line. For an averaged A-scan, the OCT B-scan is straight to the detected surface (left side in Fig. 35.6). Skin layer thicknesses can be easily identified in the averaged A-scan. For example, the epidermis layer (without stratum corneum) on the inner side of the hand is measured between the green and the blue line at $142 \mu \mathrm{m}$ (Fig. 35.6a).

On the other side, it is also possible to analyze the attenuation coefficient (Fig. 35.6b). By positioning the green and blue line to the region of interest, the averaged A-scan can be fitted in this region by a theoretical function (e.g., (35.4)).

Figure 35.6b illustrates a measured fingertip. The attenuation coefficient of the stratum corneum in this case is $\mu_{\mathrm{t}}=36 \mathrm{~cm}^{-1}$.

Measuring the thickness and the attenuation coefficient enables a quantification of structural changes of the skin.

\subsubsection{Field of View and Measurement Artifacts}

The penetration depth of the signal in the tissue is limited by several factors. The power of the light source as well as the wavelength is important. Light propagation is influenced by the medium. Soft tissue has a group index of refraction of about 1.34 similar to water [10]. Coupling of the probe beam to the skin using water or gel reduces the light reflection of the surface by index matching. Light sources in the near infrared, the so-called optical window, are preferred because both absorption by haemoglobin, melanin, and water and scattering are relatively low in this range $[11,12]$.

Figure 35.7 demonstrates a typical field of view. An overview can be given by ultrasound imaging of a larger area of skin. Twenty megahertz ultrasound has an axial resolution of about $80 \mu \mathrm{m}$. The epidermis on the upper arm (Fig. 35.7) cannot be distinguished using this method. The measurement depth of $20 \mathrm{MHz}$ ultrasound is relatively high. Therefore, the border to the subcutaneous fat is clearly seen (white arrow in Fig. 35.7a). This border is also seen in the TD-OCT image (white arrow in Fig. 35.7c). Additionally, the TD-OCT distinguishes the border between the epidermis and dermis (black arrow in Fig. 35.7c) and shows some structures like blood vessels inside the dermis. About $10 \mu \mathrm{m}$ below the surface (black arrow in Fig. 35.7b), the SDOCT distinguishes the stratum corneum. The low intense dark areas depict small structures like blood vessels inside the dermis.

Besides the high resolution imaging of the skin by OCT, there are different measurement artifacts that must also be described. Figure 35.8 shows some characteristic measurement artifacts in SD-OCT images. By measuring a hair (Fig. 35.8a), the light can be twice reflected inside the hair, and so it seems that there would be a second hair that is not true. There is a signal shadow below the hair. By measuring without an index-matched tissue surface, the entrance signal can become such high that an echo appears in double distance to the reference plane (arrow), because the non-linearity of the detector produces harmonics of the interference frequency. 

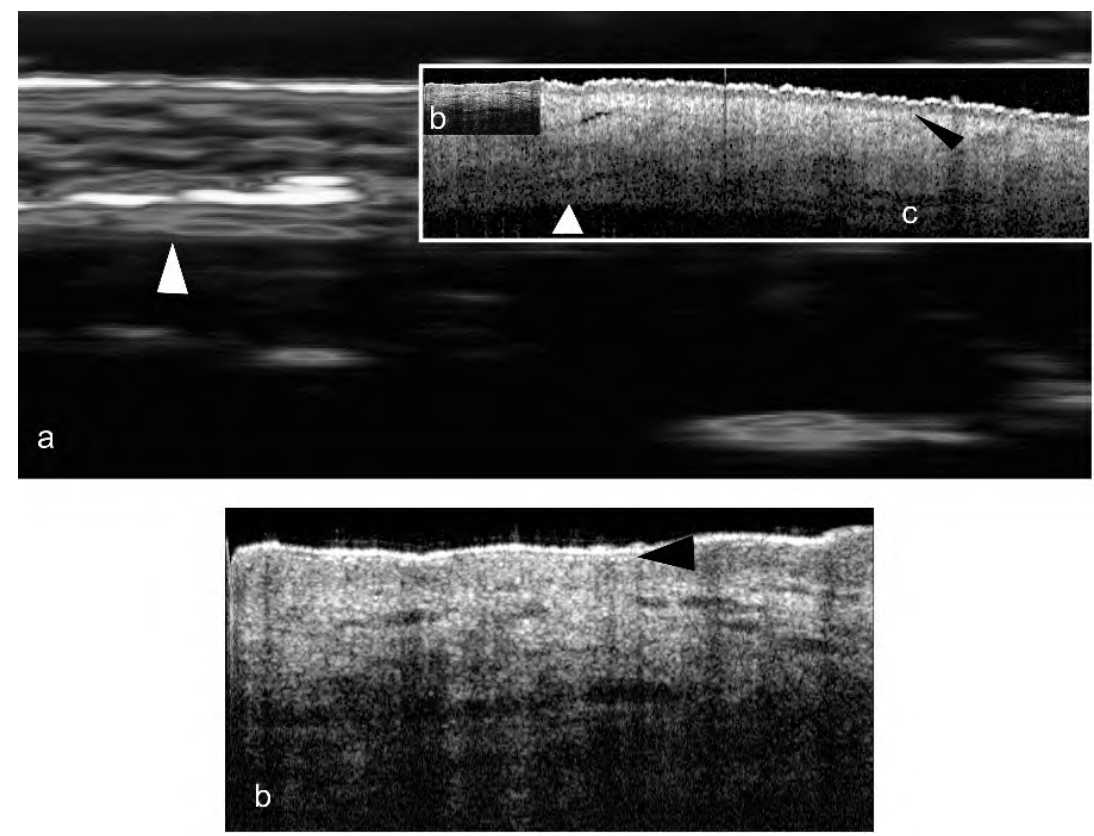

Fig. 35.7. Skin at the upper arm. (a) Large image: $20 \mathrm{MHz}$ ultrasound. (c) $1,310 \mathrm{~nm}$ TD-OCT (lateral $6 \mathrm{~mm})$. (b) $930 \mathrm{~nm}$ SD-OCT inserted into (c) and (a) $\left(1 \times 0.5 \mathrm{~mm}^{2}\right)$
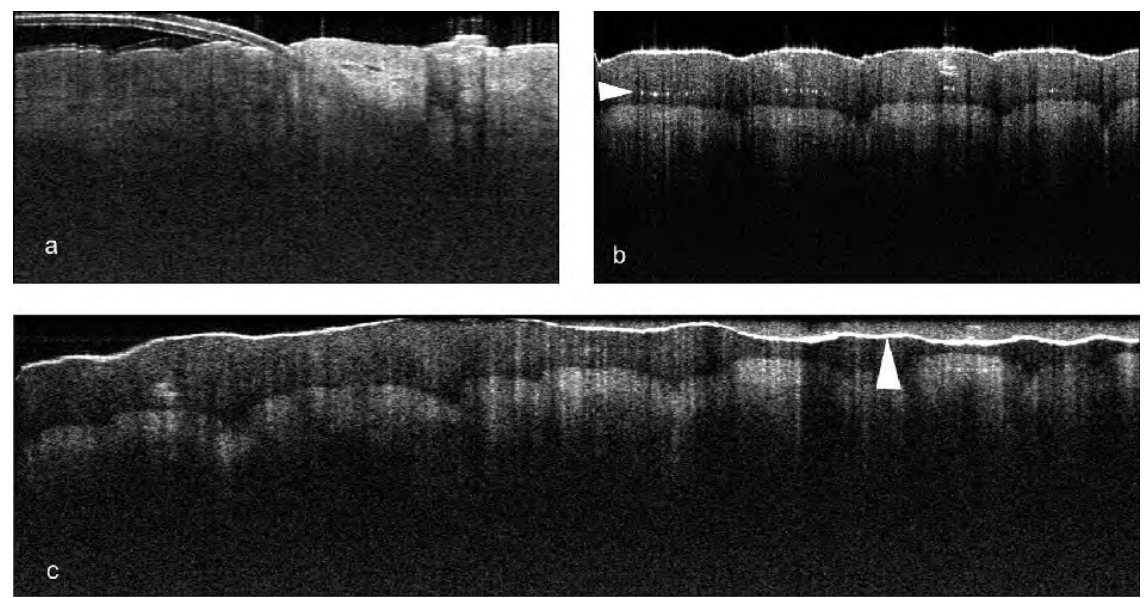

Fig. 35.8. Artefacts in SD-OCT of (a) skin with hair $\left(2 \times 1 \mathrm{~mm}^{2}\right)$, (b) fingertip with harmonic signal from the strong surface reflection $\left(2 \times 1 \mathrm{~mm}^{2}\right)$, and $(\mathbf{c})$ fingertip with folding artefact $\left(4 \times 1 \mathrm{~mm}^{2}\right)$ 
When the optical path length of the probe is too short, the signal will be folded into the measurement window (arrow in Fig. 35.8c), because SDOCT cannot discriminate between positive and negative distances from the reference plane.

\subsubsection{Functional OCT Techniques for Tissue Characterization}

In OCT, the spatial variation of the backscattered photons is the main source of contrast. Nevertheless, the probe signal is influenced by several other physical properties of the tissue. The refractive index can be determined in different depth of the tissue, providing information on the water content $[6,10,13]$. The light is changed in amplitude, phase, and polarization. This additional information might be of diagnostic value and can be extracted comparable with a tissue staining for increasing the contrast. Like in ultrasound, Doppler-OCT flow measurements can be performed by measuring the Doppler shift of light scattered from blood [14-17]. Polarization-sensitive OCT has the ability to visualize the skin birefringence, which is mainly dependent on the collagen orientation [18-24]. OCT elastography is an imaging of microscopic deformation, strain, and stiffness of tissue [25]. With all these techniques, the contrast between normal and diseased skin can be enhanced.

\subsection{Application of OCT in Dermatology}

The golden standard of morphological investigation of the skin is histological examination of tissue biopsies. Despite its invasive character, histology has several disadvantages: The surgical technique causes artifacts of the tissue, i.e., squeezing, shrinkage, collapse of vessels, and dehydration. The preparation of the probe is time-consuming and expensive. Furthermore, it represents a snapshot of the reality and is never repeatable at the same site. Hence, there is a demand for a noninvasive high-resolution imaging technique to improve diagnosis and control therapeutic efficacy. In addition, an in vivo method bears the potential to give new insight into dynamic processes, which will never be detectable by histology.

Optical coherence tomography provides two-dimensional cross-sectional invivo images of tissue like histological sections with a resolution of $15 \mu \mathrm{m}$ and less. In these images, the layered structure of the skin with epidermis, dermis, blood vessels, and skin appendages like hair follicles and glands is visible. Furthermore, pathologic conditions that are accompanied by architectural changes like tumor cell clusters or enlarged single atypical cells are detectable with this resolution. Compared to other tissues like the eye or mucous membranes, the skin is difficult to image with OCT because it is a strong scattering medium. Therefore, the imaging depth is more limited. On the other side, the skin surface is easy to reach by the applicators of the system. 


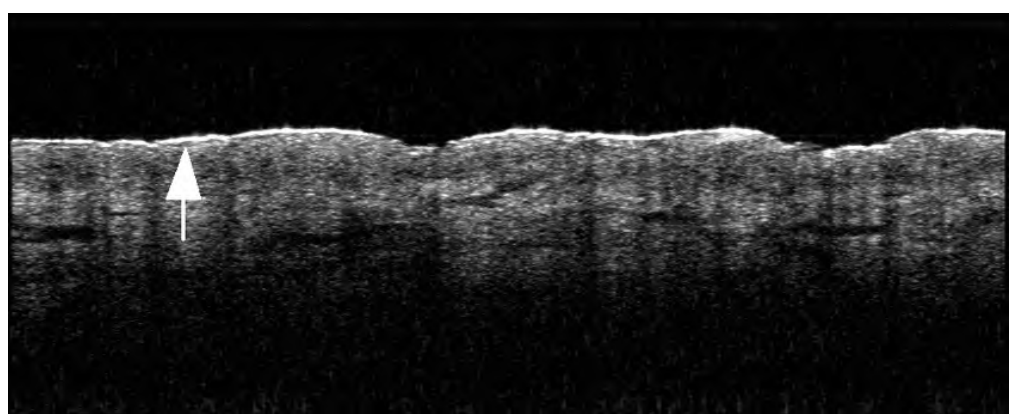

Fig. 35.9. High-resolution OCT image of healthy forearm skin. The entrance signal is splitted into two lines (arrow) corresponding to the thin stratum corneum. $2 \times$ $0.8 \mathrm{~mm}^{2}$ (OCT system provided by Thorlabs HL AG)

Dependent on the location, the visible appearance of normal healthy skin in OCT is variable. The reflection of light at the skin surface leads to a strong entrance signal due to the refractive index mismatch of the medium above the skin (normally air) compared to the skin, which has a refractive index similar to that of water. The entrance signal can be lowered by the application of gel or ointments on the surface for index matching. At ridged skin, the entrance signal is split into two lines when using high-resolution OCT (Fig. 35.9).

This thin superficial layer corresponds to the stratum corneum, which has a thickness of about $10 \mu \mathrm{m}$. The next more or less flat layer is the viable epidermis. The scattering inside the epidermis, which is composed mainly of keratinocytes, is low and homogenous. In the papillary dermis, the signal intensity increases again due to an irregular distribution of collagen bundles and capillaries. Therefore, it is mostly possible to determine the border between epidermis and dermis [26-28]. The reticular dermis shows a strong scattering with several lower reflecting structures. Hair follicles and glands can be distinguished by the characteristic shape, whereas blood and lymphatic vessels are signal free longish or round cavities. The signal intensity in deeper parts of the dermis decreases due to a limited penetration depth of the light caused by multiple scattering and absorption.

At the palms and soles, the stratum corneum is much thicker and constitutes a thick wavy homogenous layer at the surface with some spiral sweat gland ducts inside (see Figs. 35.4 and 35.13) [29]. The border between the epidermis and dermis is less demarcated because it is more toothed. The dermis is less signal intense compared to other regions of the body [30-34].

\subsubsection{Diagnosis of Skin Diseases}

\section{Skin Tumors}

Superficial parts of epithelial, mesenchymal, and melanocytic skin tumors are visible in the OCT images [34]. 


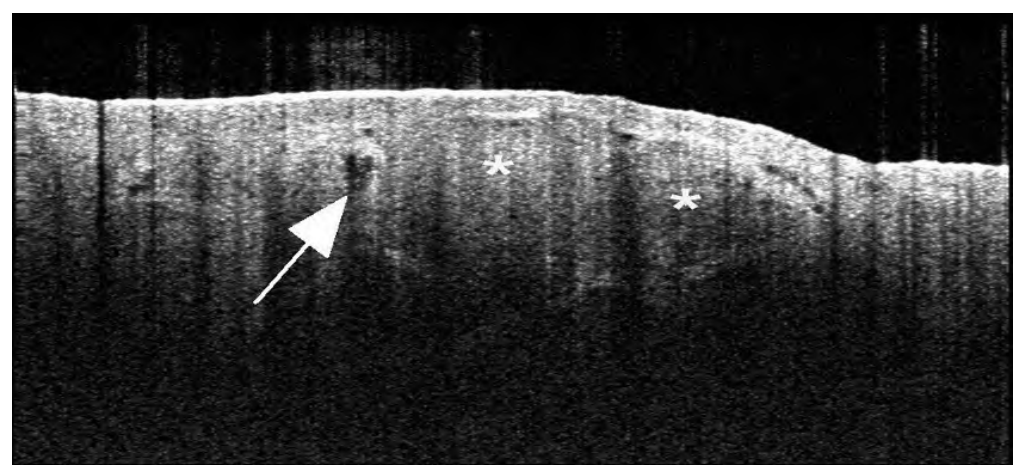

Fig. 35.10. High-resolution OCT of a basal cell carcinoma at the temple. The tumor cell aggregates are marked by stars and the dilated vessels by an arrow. $2 \times 0.9 \mathrm{~mm}^{2}$

In OCT images of epithelial tumors like basal cell carcinomas, the tumor cell aggregates have a similar appearance regarding signal density and distribution compared to the epidermis. They have often contact with and derive from the epithelium. The tumor can be distinguished from the surrounding fibrous stroma [35], in some cases with a signal poor cleft. Typically, enlarged blood vessels are seen in the upper dermis, lying above the tumor aggregates (Fig. 35.10).

Squamous cell carcinomas have a similar appearance but are in some cases more difficult to visualize because of the stronger keratinization of the tumor, which may lead to a signal reflection at the surface and a shadow below. Blood crusts induce the same artifact and should be removed carefully before imaging. The detection of the lateral tumor margins and of the lower border is possible in thin tumors, but not in thicker ones with a more iceberglike growth. Deeper tumor masses escape attention because of the limited penetration depth of the signal.

Mesenchymal tumors like fibrous histiocytoma appear as deep irregularities of the collagen distribution below an elevated, but unaffected epidermis. Depending on the degree of fibrosis or sclerosis, the signal intensity of the tumor may be stronger or lower compared to the adjacent dermis.

Melanocytic lesions are represented as strong scattering areas with a cloudy appearance in the OCT image. In general, melanin pigment increases the scattering and signal attenuation. In benign melanocytic nevi, larger nests of nevus cells are distinguishable. Morphologic changes of the epidermis like acanthosis and enlargement of the rete ridges are typical for these benign lesions and can be detected in the OCT images. In malignant melanoma, the signal distribution is less homogenous compared to benign nevi. The signal attenuation coefficient $[\mu]$ is significantly increased in the melanoma compared to the adjacent healthy skin (own unpublished data). Some strong reflecting pigmented globules of atypical melanocytes or melanphages induced a signal shadow below (Fig. 35.11). 


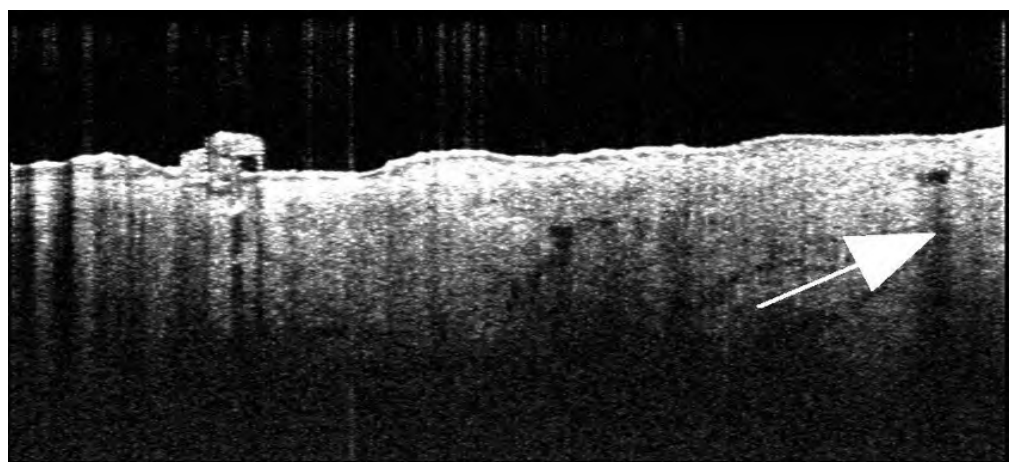

Fig. 35.11. High-resolution OCT of a malignant melanoma at the forearm. Some structures are signal rich, other induce a signal shadow below (arrow). The scattering inside the tumor is very irregular. $2 \times 0.9 \mathrm{~mm}^{2}$

In contrast to OCT images of melanocytic nevi, changes of the epidermis like ulceration, crusts, and atrophy, which are due to the malignant growth pattern of melanomas, are visible. The demarcation between epidermis and dermis is less clear due to the tumor infiltrates in the junctional zone. In most cases, the contrast between melanocytes and keratinocytes is too poor to see a pagetoid spread of melanoma cells inside the epidermis. Therefore, there might be some hints to malignancy, but a clear-cut differential diagnosis between benign and malignant melanocytic lesions is still not possible using OCT at its present resolution.

In summary, a differential diagnosis between epithelial and nonepithelial tumors is reliable using OCT, but between malignant and benign tumors of the same origin it remains difficult. Systematic studies on skin tumors using OCT are still lacking.

\section{Inflammatory Skin Diseases}

Inflammation of the skin is accompanied by specific morphologic changes of the skin known from histology. The stratum corneum shows a thickening with a hyper- and parakeratosis. The epidermis is thickened, too, due to spongiosis (intercellular edema) and proliferation of the keratinocytes. In the dermis, the water content increases as well. Additionally, cellular infiltrates composed of lymphocytes and leucocytes are typical changes.

Systematic OCT studies of inflammatory skin diseases have demonstrated that these conditions are visible and could be quantified and monitored over time using OCT. Eczematous skin was investigated using an experimentally induced irritant contact dermatitis after application of sodium lauryl sulphate as a model. It showed a thickening of the entrance signal and the epidermis as well as a dilation of vessels in the upper dermis and a decrease in light scattering due to edema [33]. In blistering diseases, the location of the cleft can be determined. Therefore, OCT allows a fast and noninvasive differential 
diagnosis between bullous autoimmune diseases like pemphigus vulgaris with an intraepidermal cleft and bullous pemphigoid as a subepidermal blistering dermatosis.

In psoriasis, a severe parakeratosis leads to a multilayered entrance signal. Pustules are seen as cavities within the epidermis, filled with inhomogenous material corresponding to microabscesses of leucocytes [9].

Wound healing can be observed in a noninvasive way using OCT [36]. The occlusion of the defect by blood crusts and the regeneration of the epithelium are detectable. The granulation tissue and the scar formation lead to characteristic changes of the signal attenuation coefficient in the upper dermis with a lowering in the first phases of wound healing and an increase at the end (Fig. 35.12).

Beside the visualization of morphologic changes in inflammatory skin diseases, OCT allows a graduation of the disease with a quantification and objective scoring of the severity and a monitoring over time in a noninvasive way.

\subsubsection{Evaluation of Treatment Effects}

Additional to the assessment of morphologic changes, OCT enables an exact determination of the thickness of layers. Therefore, this imaging method is a valuable tool to quantify treatment effects.

The skin as a target for therapeutic intervention in diseased condition is easy to reach by topical treatment. Most diseases can be treated successfully using creams and ointments containing the drug as an active component and a base formulation to improve the penetration and for skin care. Several studies using OCT were performed to investigate treatment effects. Already the base formulation, gel, cream, or ointment, has an influence on the OCT image. Application of various agents (glycerol, paraffin oil, 10\% urea in petrolatum, petrolatum, ultrasonic gel) onto the skin is followed by a decrease in the reflectivity of the surface, accompanied by a smaller entrance peak in the averaged A-scan [33]. The penetration depth of the light signal is increased due to a lower light attenuation and scattering at the surface. This unspecific effect is attributed to refractive index matching and is greatest for paraffin oil. In an animal model it could be demonstrated that application and injection of glycerol prior to OCT measurements also increase the penetration depth of the signal by reduction of random scattering and making the skin less turbid for visible wavelengths $[37,38]$.

In psoriasis, OCT studies have shown that the severely thickened epidermis nearly turned to normal after four weeks of therapy without reaching the values of the adjacent healthy skin [9]. UV irradiation induced dose dependent visible changes of the epidermis like parakeratosis and thickening, accompanied by alterations of the scattering coefficient due to erythema and pigmentation $[39,40]$. An increase of the water content in the stratum corneum by occlusion, prolonged water contact, or application of moisturizers is followed 


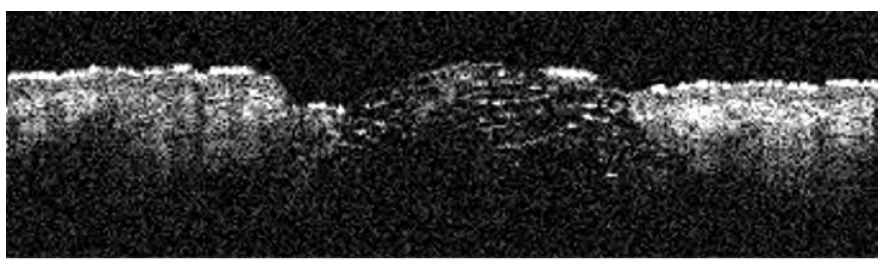

(a)

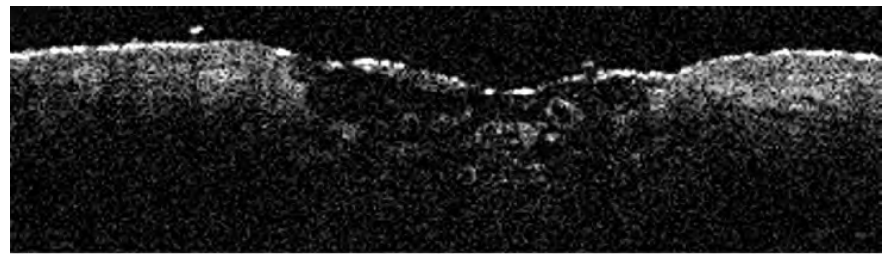

(b)

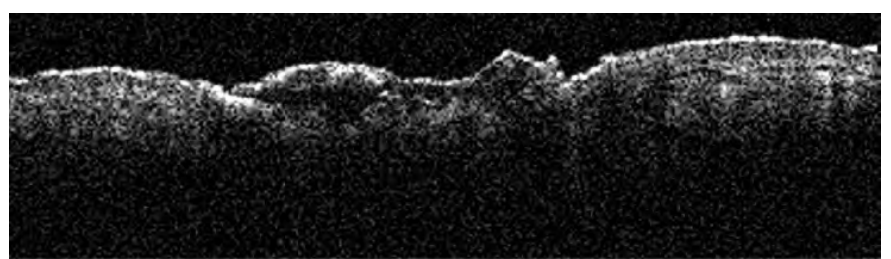

(c)

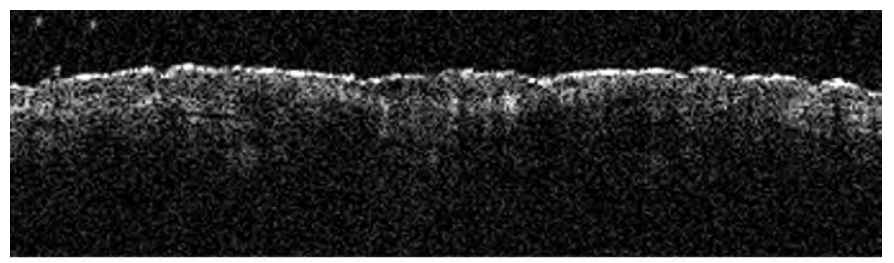

(d)

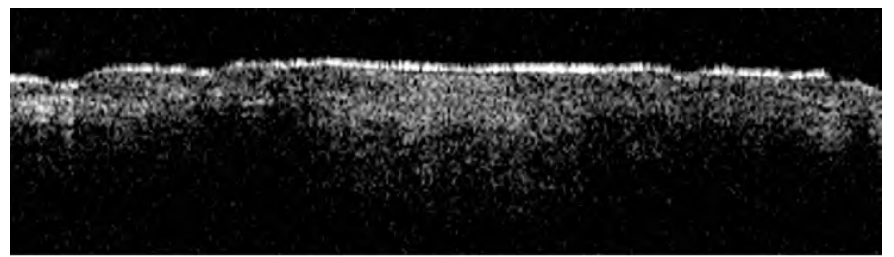

(e)

Fig. 35.12. OCT of wound healing of a $4 \mathrm{~mm}$ punch biopsy. The fresh wound (a) is covered with a crust after 4 days (b). After 4 and 13 days (c), the scattering in the upper dermis below the crust is irregular due to granulation tissue. After 22 days, the surface is closed (d). After 90 days (e), the scattering in the upper dermis is increased due to scar formation. $6 \times 1.7 \mathrm{~mm}^{2}$ 


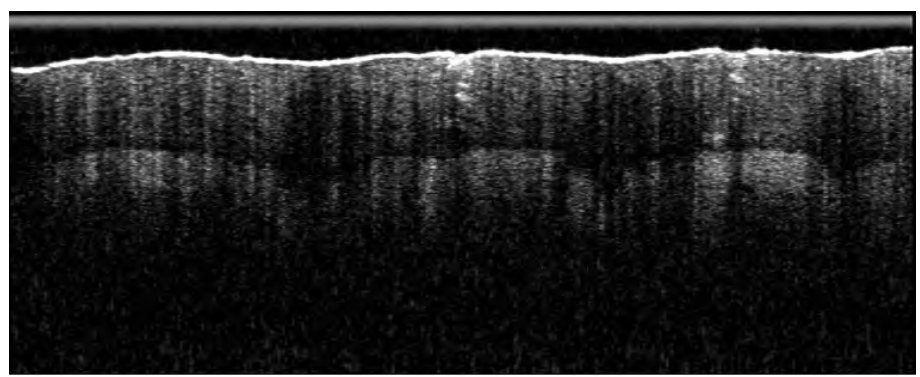

(a)

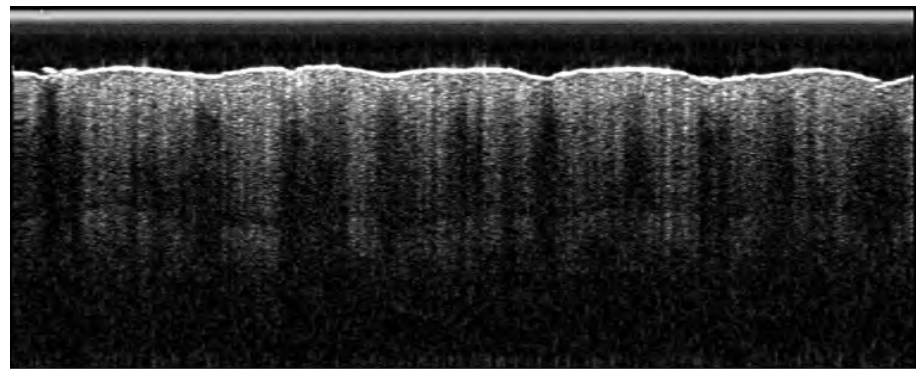

(b)

Fig. 35.13. High-resolution OCT image of healthy skin at the fingertip (a). The first thick wavy layer is the stratum corneum with some spiral sweat gland ducts. After $5 \mathrm{~h}$ of wearing gloves (b), the stratum corneum shows a swelling. The scattering of upper parts of the horny layer is increased. $2 \times 0.8 \mathrm{~mm}^{2}$

by a marked swelling, which can be quantified easily at the fingertips using OCT (Fig. 35.13). Moreover, the effect of consecutive tape stripping on the thickness of the stratum corneum can be quantified in the OCT images [33].

Skin atrophy is an important side effect of corticosteroid therapy. For monitoring the dermal thickness, high-frequency ultrasound is established as a standard method. The thickness of the epidermis is only measurable using OCT due to the higher resolution. A systematic OCT study on steroid atrophy has shown that the epidermal atrophy starts within the first days of therapy and is more pronounced than the change of dermal thickness. The degree of epidermal thinning correlates to the potency of the corticosteroids, but is observed even in the new-generation steroids with increased benefit-risk ratio [41].

\subsection{Comparison with Other Skin Imaging Methods}

\subsubsection{High-Resolution Ultrasound}

Ultrasound with a mean frequency of $20-75 \mathrm{MHz}$ is established for skin imaging since many years. In a cross-sectional field of view of about $12 \mathrm{~mm}$ length and $6 \mathrm{~mm}$ depth, the dermis appears as an echorich layer with an echopoor 
subcutaneous fat below. The resolution at $20 \mathrm{MHz}$ is between 80 and $200 \mu \mathrm{m}$. A measurement of the thickness of dermal tumors as well as a quantification of changes within the dermis and the subcutaneous fat is easily possible. It is used in dermatology as a routine method for presurgical determination of the thickness of malignant melanomas and for monitoring connective tissue diseases like scleroderma. In these images, the epidermis is not detectable. By increasing the frequency up to 100 or more megahertz, images comparable with OCT can be produced. It should be reminded that they represent acoustical and not optical properties of the tissue and that the technical expenditure at ultrahigh frequencies is much higher compared to OCT.

\subsubsection{Confocal Laser Microscopy}

In contrast to OCT and ultrasound, confocal microscopy provides a horizontal view on a focal plane with a high resolution. Further technical developments of confocal laser microscopy have led to an in vivo system for skin imaging. The horizontal field of view is a small spot of $500 \times 500 \mu \mathrm{m}^{2}$, but by lateral moving the object block images of up to $10 \times 10 \mathrm{~mm}^{2}$ can be composed. The resolution is about $1 \mu \mathrm{m}$ and much higher compared to OCT. Single cells and even cell nuclei can be distinguished. The focal plane can be moved stepwise up- and downwards, but penetration depth of the signal is generally limited to about $200 \mu \mathrm{m}$.

Confocal microscopy is an interesting method for imaging the epidermis and stratum papillare of the upper dermis with a cellular resolution, and might be of value for diagnosis of superficial skin tumors and imaging of the epidermal-dermal junction. In summary, OCT is an intermediate between both methods, high-frequency ultrasound and confocal microscopy, regarding resolution and detection depth.

\subsection{Conclusion and Outlook}

OCT is the only method for cross-sectional imaging of the human epidermis and upper dermis in vivo. In the images, a determination of the thickness of the stratum corneum and the epidermis is easily possible.

Several studies have demonstrated that it is a valuable method for monitoring inflammatory skin diseases and wound healing as well as for quantification of treatment effects like steroid atrophy.

For tumor diagnosis, OCT provides information on the architecture of a lesion. Systematic studies on the sensitivity and specificity for differential diagnosis between skin tumors are still lacking. However, the resolution and contrast are still not high enough to differentiate between benign and malignant tissue on a cellular level. On the other side, the increasing importance of nonsurgical therapeutic modalities of skin cancer requires a noninvasive method for control of healing to replace biopsies. 
The main advantage compared to conventional histologic investigation is the real-time image acquisition of $\mathrm{OCT}$, which allows a dynamic view into the skin.

Furthermore, OCT as a noninvasive method might provide new insight into physiologic or pathophysiologic processes that are dependent on the integrity of tissue.

By combining different optical methods like epiluminescence microscopy, OCT, and confocal microscopy within one system, the diagnostic value of the single methods may be improved significantly. Contrast agents or staining methods for tissue differentiation are still under investigation.

The field of high-resolution optical imaging methods is rapidly developing. Therefore, recent advances of OCT technology as well as the aforementioned functional techniques for tissue characterization might lead to new applications in dermatology in the near future.

\section{References}

1. B. Golubovic et al., Opt. Lett. 22, 1704 (1997)

2. S.R. Chinn, E.A. Swanson, J.G. Fujimoto, Opt. Lett. 22, 340 (1997)

3. J.G. Fujimoto, Nat. Biotechnol. 21, 1361 (2003)

4. W. Drexler, J. Biomed. Opt. 9, 47 (2004)

5. A. Unterhuber et al., Phys. Med. Biol. 49, 1235 (2004)

6. A. Knüttel, S. Bonev, W. Knaak, J. Biomed. Opt. 9, 265 (2004)

7. D.J. Faber et al., Opt. Exp. 12(19), 4353 (2004)

8. E. Lankenau, Optische Kohärenztomographie: Dispersive Einflüsse und Anwendungen in der medizinischen Diagnostik. Berlin: http://www.dissertation.de -Verlag im Internet GmbH, ISBN 3-89825-674-X, (2003)

9. J. Welzel, M. Bruhns, H.H.Wolff, Arch. Dermatol. Res. 295, 50 (2003)

10. G.T. Tearney et al., Opt. Lett. 20, 2258 (1995)

11. A.W. Sainter, T.A. King, M.R. Dickinson, J. Biomed. Opt. 9, 193 (2004)

12. T.L. Troy, S.N. Thennadil, J. Biomed. Opt. 6, 167 (2001)

13. A. Knüttel, M. Boehlau-Godau, J. Biomed. Opt. 5, 83 (2000)

14. B.H. Park et al., Opt. Exp. 11, 782 (2003)

15. V.X.D. Yang et al., Rev. Sci. Instrum. 74, 437 (2003)

16. J. Kehlet Barton et al., Dermatology 198, 355 (1999)

17. S.J. Tang et al., Gastrointest. Endosc. 58, 591 (2003)

18. M.C. Pierce et al., J. Invest. Dermatol. 123, 458 (2004)

19. J. Strasswimmer et al., J. Biomed. Opt. 9, 292 (2004)

20. M.C. Pierce et al., J. Biomed. Opt. 9, 287 (2004)

21. M. Pircher et al., Opt. Exp. 12, 3236 (2004)

22. J.F. de Boer et al., Opt. Exp. 3, 212 (1998)

23. S.M. Srinivas et al., J. Biomed. Opt. 9, 207 (2004)

24. B.E. Applegate et al., Opt. Lett. 29, 2252 (2004)

25. J.M. Schmitt, Opt. Exp. 3, 199 (1998)

26. J. Weissman, T. Hancewicz, P. Kaplan, Opt. Exp. 12, 5760 (2004)

27. T. Gambichler et al., J. Biomed. Opt. 10, 44008 (2005)

28. S. Neerken et al., J. Biomed. Opt. 9, 274 (2004) 
29. H. Fruhstorfer et al., Clin. Anat. 13, 429 (2000)

30. J. Welzel et al., J. Am. Acad. Dermatol. 37, 958 (1997)

31. A. Pagnoni et al., Skin Res. Technol. 5, 83 (1999)

32. N.D. Gladova et al., Skin Res. Technol. 6, 6 (2000)

33. J. Welzel et al., Br. J. Dermatol. 150, 220 (2004)

34. J. Welzel, Skin Res. Technol. 7, 1 (2001)

35. F.G. Bechara et al., Skin Res. Technol. 10, 169 (2004)

36. A.T. Yeh et al., J. Biomed. Opt. 9, 248 (2004)

37. G. Vargas et al., Lasers Surg. Med. 24, 133 (1999)

38. G. Vargas et al., Photochem. Photobiol. 77, 541 (2003)

39. T. Gambichler et al., Clin. Exp. Dermatol. 30, 79 (2005)

40. T. Gambichler et al., Arch. Dermatol. Res. 297, 218 (2005)

41. M. Cossmann, J. Welzel, Br. J. Dermatol. 155, 700 (2006) 